\title{
LA TEORÍA DE LOS CÓDIGOS Desigualdades lingüísticas en educación
}

\author{
THE NEW REALITY OF CODE THEORY \\ The existence and growth of linguistic inequalities \\ in the world of education
}

\begin{tabular}{|c|c|}
\hline $\begin{array}{r}\text { DOLORS MA } \\
\text { Universid }\end{array}$ & $\begin{array}{l}\text { ORAL ARQUÉ } \\
\text { de Lleida }\end{array}$ \\
\hline $\begin{array}{l}\text { PALABRAS CLAVE ADICIONALES } \\
\text { Desigualdades educativas, Capital cultural, } \\
\text { Subculturas, Clase social. }\end{array}$ & $\begin{array}{l}\text { ADDITIONAL KEYWORDS } \\
\text { Educational Inequalities, Cultural Capital, } \\
\text { Subcultures, Social Class. }\end{array}$ \\
\hline
\end{tabular}

RESUMEN. Las desigualdades lingüísticas en educación constituyen un problema que puede acentuarse con el cambio de sociedad. Este artículo recupera la teoría de los códigos de Basil Bernstein desde una perspectiva culturalista para aclarar sus aspectos más polémicos $\mathrm{y}$, al mismo tiempo, introducir un nuevo modelo de análisis que pone en relación el capital cultural de las familias con el nivel de desarrollo lingüístico de los adolescentes. Se observa que el nivel de estudios alcanzado por los padres adquiere una notable importancia en el desarrollo de la competencia comunicativa, especialmente en el desarrollo de las habilidades correspondientes al lenguaje escrito en los adolescentes de primer curso de Educación Secundaria Obligatoria.

\begin{abstract}
Linguistic inequalities in education do not only represent a hitherto unresolved problem but may even be exacerbated by social change (synonymous with technological and multicultural societies). In this article we have re-examined Basil Bernstein's code theory from a culturalist perspective and tried to clarify some of most controversial features and to present a new analytical model that relates the cultural capital of families to the level of linguistic development in adolescents. We observed that the level of studies reached by parents has a notable bearing upon the development of communicative competence, and particularly upon the development of written language skills, in adolescents studying the first course of Compulsory Secondary Education.
\end{abstract}

E-mail: dmayoral@geosoc.udl.es

Revista Internacional de Sociología (RIS)

Tercera Época, $\mathrm{N}^{\circ} 41$, Mayo-Agosto, 2005, pp. 109-135. 


\section{INTRODUCCIÓN}

Uno de los temas centrales en la Sociología de la Educación ha sido y es la influencia de las desigualdades sociales en el logro educativo. En este sentido, uno de los aspectos que menor atención ha recibido ha sido el análisis del desarrollo intelectual relacionado con variables de carácter estrictamente sociológico. ¿En qué medida el desarrollo de las capacidades cognitivas se encuentran vinculadas a la localización de posiciones ventajosas en la estructura social?, ¿cómo influye esta posición en la creación de unos hábitos sociales de razonamiento susceptibles de transformarse en cualidades intelectivas individuales con carácter diferenciador?, ¿cuál es el peso del capital cultural de los padres en el éxito escolar y profesional de los hijos?, ¿hay que dar por sentado que la familia influye poco en dicho desarrollo cognitivo?, ¿constituyen los tests una auténtica medida de las capacidades intelectuales de los individuos?, ¿cómo detectamos el nivel de partida de los chicos/as cuando se integran en el contexto educativo?, ¿hasta qué punto la escuela tiene la capacidad de corregir desigualdades de origen familiar? Y si la tiene, ¿cómo se explica el mantenimiento de las desigualdades sociales?. Éstas, entre otras muchas preguntas, no han recibido todavía una explicación suficientemente satisfactoria.

A pesar de la significativa contribución de Bernstein a la Sociología del desarrollo cognitivo y la importancia de los procesos que se desarrollan en los hogares, este aspecto ha permanecido como un problema periférico. Las estructuras de clase social que ponen en juego toda una serie de recursos en educación, capital cultural y oportunidades sociales pueden reconocerse como causas efectivas de las diferencias cognitivas, de los hábitos de razonamiento. Por este motivo, los chicos/as de clase media realizan mejores progresos en el sistema educativo que los chicos pertenecientes a la clase obrera. ¿Podemos vincular las desigualdades cognitivas a las desigualdades lingüísticas?, ¿qué influencia tiene la localización en la estructura social en el desarrollo de este tipo de desigualdades?, ¿cuál es el efecto del capital cultural en el desarrollo de las desigualdades lingüísticas?, ¿qué consideración han merecido las desigualdades lingüísticas en educación?

La consideración de las desigualdades lingüísticas en el sistema educativo ha sido más bien escasa. Bernstein, el autor que planteó este concepto, ha recibido un trato desigual desde las diferentes disciplinas y ha tenido nula repercusión en las prácticas docentes. En este artículo se pretende recuperar dicho concepto como un concepto clave que nos ayude a explicar por qué determinados grupos de población obtienen elevadas tasas de fracaso escolar. Creemos que la teoría bernsteniana de los códigos lingüísticos debe ser recuperada en un nuevo planteamiento que posibilite una mejor comprensión. A pesar del desuso en el que ha caído esta teoría en las dos últimas décadas, las desigualdades lingüísticas se manifiestan hoy con más fuerza que nunca puesto que, no solamente pueden ser vistas como el resultado de determinados efectos de clase social, sino también 
como consecuencia de la aparición de nuevos modelos de comunicación que se han extendido entre los jóvenes a partir del uso de las tecnologías de la información y de la comunicación. Nuestro análisis va a centrarse en la pertinencia del concepto desigualdades lingüisticas y su relación con el contexto escolar a partir del concepto de código lingüístico formulado por Bernstein. Además, vamos a analizar el peso de las variables capital cultural y clase de ocupación de los padres en el desarrollo lingüístico-cognitivo de los/as adolescentes. Sin embargo, antes de entrar en consideraciones sobre el análisis, queremos precisar qué entendemos por desigualdades lingüísticas y cuándo es pertinente hablar de la existencia de este tipo de desigualdades.

Las desigualdades lingüísticas son las que se fundamentan en la posesión de una competencia lingüística escasamente adecuada $o$, sencillamente, no adecuada a determinados contextos previamente establecidos y categorizados de carácter evaluativo. Evidentemente, hablamos de contextos evaluativos (si no hay evaluación —entendida en términos generales - no hay desigualdades); queremos decir que es extremadamente dificil hablar de desigualdades lingüísticas en determinados contextos de la vida cotidiana ya que, si no es por causas orgánicas, todos los hablantes poseen la competencia suficiente para desarrollarse en sus contextos naturales como pueden ser la familia, la comunidad o el barrio de residencia.

Dichas desigualdades aparecen cuando los individuos puestos en un contexto determinado de carácter evaluativo pueden presentar insuficiencias en la comunicación, ya sea en la comprensión, o ya sea en la misma expresión verbal o escrita. Obviamente, esta definición tiene un carácter extensivo a problemáticas diferentes como pueden ser la inadecuación de la competencia lingüística de un niño al contexto educativo, o bien la inadecuación lingüística de un inmigrante $a$ un contexto laboral en el que se le exige una competencia en una segunda o tercera lengua. Por otra parte, el concepto de desigualdad lingüística no puede ser aplicado como el resultado del no conocimiento de un registro determinado -imaginemos el registro propio de una profesión, puesto que se supone que para acceder a esta profesión se han realizado unos estudios que preparan para el conocimiento de este registro-. Además, el dominio de un dialecto no ha de ser causa de desigualdad lingüística, aunque el uso de términos procedentes de determinados dialectos no aceptados normativamente puedan llegar a ser motivo de sanción, muy especialmente en el contexto escolar.

En definitiva, solamente es pertinente hablar de desigualdades lingüísticas en determinados contextos y valga esto como réplica a las numerosas críticas realizadas por Stubbs (1982) y Lavob (1979), por poner unos ejemplos. Dichos sociolingüistas no acabaron de captar el problema en sus dimensiones sociológica y educativa. Ello, no obstante el anterior comentario, no justifica las dificultades metodológicas que tuvo Bernstein en su proceso de investigación y en la misma formulación del concepto código, así como tampoco se pretende minimizar el problema de las desigualdades lingüísticas que puedan tener otro origen. 


\section{LA TRIPLE PROBLEMÁTICA DE LA TEORÍA DE LOS CÓDIGOS: UNA SÍNTESIS}

La formulación inicial realizada por Bernstein que dará lugar a la teoría de los códigos lingüísticos no ha estado exenta de polémica. La teoría del déficit lingüístico, la formulación de dos modos de habla según el tipo de lenguaje-uso y el concepto de código lingüístico propiamente dicho han sido puestos en cuestión en numerosas ocasiones. Bernstein desarrolla la teoría del déficit lingüístico, aunque con matices, tal como el mismo autor pone de relieve en un artículo crítico sobre la educación compensatoria. Para este autor, es improcedente hablar de educación compensatoria si previamente no se ha ofrecido a los niños un entorno educativo adecuado (Bernstein, 198:195). Según el mismo Bernstein, "el niño pierde a las puertas de la escuela, de la misma manera que sus padres, su identidad social, su modo de vida y su representación simbólica". Tanto el concepto de educación compensatoria como el de déficit lingüístico impiden, en palabras de Bernstein (198:196-197), centrar la atención en las deficiencias de la propia escuela para centrarse en las deficiencias de la propia comunidad, de la familia o del mismo niño.

Por otra parte, Bernstein también establece dos modos de usar el lenguaje, que se corresponden con dos modos diferentes de relacionarse con la realidad: el lenguaje elaborado o formal y el lenguaje restringido o público. El lenguaje elaborado se relaciona con categorías cognitivas abstractas, goza de más prestigio, sus expresiones son matizadas y bien entrelazadas y constituyen el reflejo de la elaboración de la propia subjetividad. Por el contrario, el lenguaje público se vincula a expresiones cognitivas y lingüísticas más simples, menos elaboradas, más repetitivas, menos subjetivas y más comunes. Sin embargo, ello no debe inducirnos al error de creer que Bernstein menosprecia el lenguaje público tal y como se muestra en la cita posterior:

"El lenguaje público tiene su propia estética, una simplicidad y una franqueza en la expresión, es emocionalmente viril, sentencioso y poderoso y una variedad metafórica de considerable fuerza y conveniencia. Algunos ejemplos tomados de las escuelas de nuestro país tienen una belleza que muchos autores envidiarian. Es un lenguaje que simboliza una tradición y una forma de relación social en la que el individuo es considerado como un fin, no como un medio para un fin. Sustituir simplemente el lenguaje formal (que no es necesariamente lógico, impersonal, emocionalmente visceral) es separar al individuo de sus relaciones tradicionales y quizás alienarle de ellas" (Bernstein, 1989:67).

El problema se sitúa en el ámbito escolar puesto que se penaliza a los niños/as cuando su lenguaje no se corresponde con el modelo estándar —es decir, el lenguaje elaborado- Conviene destacar que esta penalización expresada a través de numerosas sanciones puede llegar a ser grave, ya que puede condicionar el éxito o el fracaso escolar. 
Por último, en la expresión del concepto "código" se evidencian, definitivamente, numerosas dificultades empíricas de algunas de sus investigaciones, muy especialmente, las que tienen el análisis del lenguaje como objeto de estudio. A la propia complejidad de una investigación que pretende poner en relación la estructura social con un determinado uso del lenguaje se le añaden los dos siguientes factores: en primer lugar, muchas de las muestras recogidas por Bernstein son de carácter oral (aparte de los tests, las matrices de Raven y la Escala de Vocabulario Forma 1 de Mill Hill) y, en segundo lugar, la población objeto de estudio ha sido reducida y podría considerarse poco representativa. Es evidente que estas críticas no suponen un rechazo del lenguaje oral. En todo caso, es necesario realizar unas matizaciones. Donde más se evidencian los distintos tipos de lenguaje-uso es en el contexto escolar. Esto nos debe hacer caer en la cuenta de que el sistema educativo se fundamenta más en el desarrollo y en la evaluación del lenguaje escrito, tal como el mismo Bernstein reconoce en una de sus últimas publicaciones ${ }^{1}$.

Así las cosas, ¿puede considerarse el análisis de la forma oral poco adecuado al tipo de investigación realizado por Bernstein? Es evidente que las respuestas categóricas no existen, aunque debemos considerar que el lenguaje oral es mucho más laxo y más desorganizado que el escrito $\mathrm{y}$, por tanto, mucho más repetitivo e inacabado. Es frecuente producir muchas secuencias unidas por conjunciones de coordinación con incluso dificultades para mantener un determinado grado de coherencia en el discurso. Quizás las formas inherentes al lenguaje oral sean menos adecuadas que las formas inherentes al lenguaje escrito si tomamos como referencia el contexto escolar. La pregunta es: si se hubiese efectuado el análisis a partir de la elaboración de un documento escrito, ¿hubiesen variado los resultados? No sabemos con certeza hasta qué punto el uso de un sistema u otro hubiese sido más riguroso; ahora bien, lo que sí sabemos es que en el contexto educativo el estatus del lenguaje escrito es muy diferente frente al del lenguaje oral. Por otra parte, el lenguaje escrito fuerza al individuo a organizarse mentalmente $y$, en consecuencia, puede mostrar con más claridad la medida de sus posibilidades lingüisticas como cognitivas 2 .

\footnotetext{
${ }^{1}$ Bernstein, B. (1993), La estructura del discurso pedagógico, Madrid, Morata.

${ }^{2}$ Según Vygostki, desde el punto de vista psicológico, la escritura no consiste en aplicar al papel funciones verbales anteriormente desarrolladas o al lenguaje oral, sino en crear sistemas psicológicos nuevos que no surgen de manera espontánea y que solamente son posibles gracias a una enseñanza sistémica. En este sentido, el dominio de la lectura y de la escritura abre el camino hacia una forma de conciencia superior (Kozulin, 1994: 181). Olson esboza el argumento sobre la influencia de la escritura sobre la cognición y habla de la superioridad de ésta sobre el lenguaje oral (citado por Kozulin, A. (1994), La psicología de Vygostky, Alianza, pp. 182). Asimismo, Simone habla del tipo de inteligencia que se desarrolla con la lectura y la escritura: quien la utiliza ha de proceder ordenadamente, por medio de pasos consecutivos y codificar sus pensamientos estableciendo diferentes jerarquías entre operaciones de diferente nivel de importancia. El paso de una inteligencia simultánea (basada en la imagen) a una inteligencia secuencial (basada en la lectura y escritura) implica una transformación profunda del estilo cognitivo (Simone, 2000: 89 y ss.).
} 
Cabe recordar también que el concepto de código va mucho más allá de la estricta consideración de la estructura lingüistica. Son correlatos de dicha estructura los significados relevantes de un determinado contexto, así como el tipo de acción que de él se deriva, con lo cual, el concepto de código vincula tres dimensiones: una dimensión cultural (efecto de la estructura social), una dimensión conductual (efecto de la coerción de la estructura sobre la acción de los individuos) y una dimensión lingüística (efecto de la estructura de poder: significados relevantes o no relevantes y su producción en una estructura lingüística determinada).

Por último, otro de los problemas que se le achacan a la teoría de los códigos de Bernstein hace referencia al concepto de clase social excesivamente impreciso y confuso que utiliza. Bernstein, en el transcurso de sus investigaciones utiliza indiferenciadamente los términos "clase" o "estatus" para referirse a la clase obrera que, en algunas ocasiones, se confunde con el de la vieja clase media. Por lo tanto, es poco preciso en la definición de este grupo. Sin embargo, plantea con bastante acierto la diferenciación entre la vieja clase media y la nueva clase media, y es precisamente en esta aportación donde reside su originalidad. Bernstein atribuye a la "nueva clase media" un modo de vida y estrategias definitorios de un modelo pedagógico que rige los principios sobre los que se edifica el sistema educativo en el último período del siglo $\mathrm{XX}^{3}$.

\section{LENGUA Y CLASE SOCIAL}

Al elaborar la teoría de los códigos, Bernstein parte de un marco de análisis anterior donde se establecen dos categorías para el análisis lingüístico: una es la que corresponde al lenguaje público y la otra es la que se corresponde con el lenguaje formal. A cada una de estas categorías les atribuye un tipo de lenguaje-uso diferente. El problema no se encuentra en la categorización en sí misma, sino que a cada una de las categorías se le atribuye un prestigio social diferente, y mientras que una es propia de contextos amicales y/o comunitarios, la otra es más propia de contextos más formales como el educativo.

Ésta es una lectura relativamente lineal de los hechos. En efecto, la competencia comunicativa es más que esto, especialmente, si se analiza desde una perspectiva sociológica. La primera pregunta que nos hemos de plantear es por qué una variedad lingüística predomina sobre la otra. La segunda es por qué, en el contexto escolar, se valora casi exclusivamente la posesión de la variedad que corresponde al lenguaje elaborado y por qué los hablantes que utilizan el lenguaje público son negativamente estigmatizados y sancionados por este hecho.

\footnotetext{
${ }^{3}$ Esta argumentación se encuentra en el segundo volumen. Bernstein establece un modelo de relación entre determinadas concepciones pedagógicas - muy especialmente, la denominada pedagogía invisible- con un tipo de ideología muy propia de las nuevas clases medias, es decir, aquellas que desarrollan su actividad en los ámbitos de la producción, de la reproducción cultural y del control simbólico de la sociedad.
} 
Bourdieu, en ¿Qué significa hablar? (1985) plantea un análisis sumamente interesante que intenta responder desde una perspectiva crítica a por qué se atribuye más prestigio a una variedad lingüística que a otra. Este autor vincula determinados estilos (usos) lingüísticos a determinadas clases sociales. También plantea el hecho de que la lengua puede considerarse como un instrumento de poder y de dominación, dado que las diferentes variedades lingüísticas se encuentran vinculadas a jerarquías o grupos sociales diferentes. Dice Bourdieu (1985) que sólo se acepta como oficial la variedad correspondiente a determinados usos burgueses, con lo cual quedan vinculados de una manera implícita: una clase social con un determinado uso del habla. Dado que esta clase es la que tiene el poder político y económico en la sociedad, inmediatamente, el tipo de lenguaje-uso que utiliza queda determinado como la variedad de prestigio, la "oficial", tal como se muestra en el siguiente texto:

\begin{abstract}
"Dicho con otras palabras, las diferencias reveladas por la confrontación de las hablas no se reducen a las que el lingüista construye en función de su propio criterio de pertinencia(...).

Los usos sociales de la lengua deben su valor propiamente social al hecho de que tales usos tienden a organizarse en sistemas de diferencias que reproducen en el orden simbólico de las separaciones diferenciales el sistema de las diferencias sociales. Hablar es apropiarse de uno u otro de los estilos expresivos ya constituidos en y por el uso, y objetivamente caracterizados por su posición en una jerarquía de estilos que expresa la jerarquía de los correspondientes grupos. Estos estilos, sistemas de diferencias clasificados y clasificantes, jerarquizados y jerarquizantes, dejan su huella en quienes se los apropian y la estilística espontánea, provista de su sentido práctico de las equivalencias entre ambos órdenes de diferencias, expresa clases sociales a través de las clases de índices estilísticos". (Bourdieu, 1985: 28).
\end{abstract}

Por tanto, según se desprende de la afirmación anterior, el peso de los factores sociales es especialmente significativo en la explicación de los hechos lingüísticos. Detrás del tipo de competencia comunicativa subyacen factores que son el origen de determinadas situaciones de desigualdad. Esto se hace evidente en el análisis de la dinámica de los sistemas educativos, de los sistemas de premios y sanciones relacionados con la competencia comunicativa $;$ y con lo que en el ámbito escolar

\footnotetext{
${ }^{4}$ Según Bourdieu (1982), éste no es necesariamente un efecto decididamente querido por los actores comprometidos con las luchas literarias y de la conservación de la lengua .... Pero, de todas maneras, donde se lleva a cabo la aplicación de la norma es en el ámbito escolar y es ahí donde se establece definitivamente la deslegitimación de aquella variedad que no se corresponde con la norma, "y que se relaciona también con la existencia de una institución como el sistema de enseñanza que, comisionado para sancionar, en nombre de la gramática, los productos heréticos, y para inculcar la norma explícita que contrapesa los efectos de las leyes de evolución, contribuye en gran medida a constituir como tales los usos dominados de la lengua consagrando el uso dominante como único legítimo, por el solo hecho de inculcarlo". (Bourdieu, 1985:34).
} 
RIS

se denomina capacidad intelectual, que constituye una muestra de la capacidad o conocimientos culturales de los individuos.

Continuando con esta argumentación, Bernstein, en Clases, Códigos y control. Estudios teóricos para una sociología del lenguaje (1989), intenta mostrar la vinculación de diferentes formas de lenguaje hablado con la organización de grupos sociales particulares 5 . Según este autor, se pueden distinguir grupos de estatus según su habla, entre los cuales la diferencia más grande se produce entre la clase media y el segmento más bajo de la clase obrera.

"Las diferencias lingüísticas, distintas de las dialécticas, tienen lugar en el entorno social normal y se pueden distinguir grupos de estatus según sus formas de habla. Esta diferencia está más marcada cuando la distancia entre niveles es muy grande. Se han hecho muchos estudios con el objeto de medir esta diferencia. Sugiero que las diferencias inter-estatus medibles a través de la facilidad lingüística provienen de los diferentes modos de habla que se dan entre la clase media y la clase obrera más baja" (Bernstein, 1989:69).

\section{LENGUA E INTELIGENCIA}

En un segundo paso, Bernstein intenta demostrar cómo el modo de expresión de la inteligencia puede ser el resultado de un aprendizaje que se produce a través de las formas de habla. Es decir, aprendiendo a hablar, el niño o la niña aprenden una manera determinada de organizar el mundo que le rodea y los significados -culturales - que son propios de contexto en el que se desarrolla y crece. En este sentido, son muy reveladoras las afirmaciones de Berger y Luckmann realizadas en La construcción social de la realidad (1988):

"Mentalmente capto la realidad de la vida cotidiana como una realidad ordenada. Sus fenómenos están organizados de antemano según unas pautas que parecen independientes de mi aprehensión y que se le imponen. La realidad de la vida cotidiana aparece ya objetificada; es decir, constituida por un orden de objetos designados como tales objetos antes de mi entrada en escena. El lenguaje empleado en la vida cotidiana me va proporcionando las objetificaciones necesarias, y postula el orden dentro del cual tienen sentido, y dentro del cual la vida cotidiana se me hace significativa (...) En este sentido, el lenguaje fija las coordenadas de mi vida dentro de la sociedad, y llena esta vida de objetos significativos" (Berger y Luckmann, 1988: 41).

${ }^{5}$ Bourdieu (1982: 53) dice que las leyes de transmisión del capital lingüístico son un caso particular de las leyes de transmisión de capital cultural transmitido de generación en generación. Según este autor, la competencia lingüística, hablando en términos escolares, depende del nivel de instrucción y de la trayectoria social. Ambos factores son absolutamente determinantes en el desarrollo de la competencia lingüística (Bourdieu, 1982: 53). 
Bernstein, en el postulado anterior, pone en cuestión el mismo concepto de inteligencia. Tradicionalmente, desde la perspectiva psicológica se ha entendido la inteligencia como una capacidad abstracta que facilitaba una determinada relación o manipulación de los objetos de la realidad. Para Bernstein, la inteligencia es una capacidad, fundamentalmente cultural, de adquisición de significados sociales. El problema se halla en que los significados sociales y/o culturales no se encuentran homogéneamente repartidos, y asimismo lo consideran Berger y Luckmann cuando hablan de la cultura como un repertorio social de conocimientos con su propia estructura de pertinencia. Según estos autores, el conocimiento se encuentra socialmente distribuido, en el sentido en que no es uniformemente poseído por los individuos o categorías de individuos, y lo que es significativo en un grupo social puede no serlo en otro (Berger y Luckmann, 1988: 70-71). Esto significa que un mismo contenido cultural puede participar de diferentes niveles de aprehensión del significado, que pueden ir desde el muy significativo al poco o nada significativo para cada uno de los grupos sociales.

La distribución desigual de significados sociales nos remite, por un lado, a un análisis de la distribución social del conocimiento en función de los diferentes grupos sociales y, en consecuencia, a la misma definición de cultura oficial. Por otra parte, nos conduce al análisis de las subculturas, al tipo de relación que determinadas subculturas mantienen con las clases sociales bajas y a la distancia entre los valores, normas y conocimientos de estas subculturas y los de la cultura oficial, centrándonos muy especialmente en el tipo de contenidos que se transmite en el sistema educativo.

\section{LA DISTRIBUCIÓN SOCIAL DE LOS SIGNIFICADOS: UNA CUESTIÓN DE CLASE}

En La Distinçión (1979), Bourdieu analiza de manera muy pertinente la relación que mantienen los diferentes grupos sociales con diferentes tipos de conocimientos y de apreciaciones culturales y sus efectos. Para empezar debemos decir que, según Bourdieu, el capital cultural tiene un efecto de asignación de estatus positivo o negativo. Este capital cultural, determinado por la herencia familiar y/o por su adquisición a través de las instituciones educativas, y legitimado por medio de diplomas o certificados escolares, tiene un efecto de posicionamiento social que se ejerce principalmente por mediación de la imagen social de la posición considerada y del futuro que esta posición, supuestamente, ofrece. No obstante, según este autor, la titulación académica no es sólo la condición evidente, el requisito de acceso a la cultura legítima. La titulación académica oculta la verdadera relación entre el capital económico y la cultura oficial, relación que se establece por medio de las condiciones que impone el contexto académico y la acción cultural de la familia, llegando a cerrar el círculo de la reproducción cultural: 
"Mediante la titulación académica lo que se designa son ciertas condiciones de existencia, aquéllas que constituyen la condición de la adquisición del título y también de la disposición estética, siendo el título el más rigurosamente exigido de entre todos los derechos de entrada que impone, siempre de manera tácita, el universo de la cultura legítima; anticipándonos a su demostración, puede afirmarse, simplificando, que las titulaciones académicas aparecen como una garantía de la aptitud para adoptar la posición estética porque están ligadas a un origen burgués o a un modo de existencia casi burguesa, que llevan aparejados un aprendizaje escolar prolongado, o bien, como es el caso más frecuente, a estas dos propiedades juntas" (Bourdieu, 1979:26).

Por otro lado, Willis, en Aprendiendo a trabajar (1988), nos ofrece otra perspectiva de la relación que se establece entre la cultura oficial y las subculturas vinculadas a la clase obrera. En este caso, el análisis se centra en cómo determinadas formas culturales de la clase obrera penetran en las nuevas generaciones, produciendo un efecto no deseado de reproducción cultural a través de la participación activa y creación constante de nuevas formas culturales por parte de los individuos que pertenecen a esta misma clase. En este caso, los individuos mantienen una tensión dialéctica entre las propias formas y las formas de la cultura legítima que representa la escuela. Así pues, el rechazo hacia el sistema educativo se centra en una concepción pragmática que los lleva a considerar la escuela como "no útil" y escasamente significativa para el propio desarrollo individual que se refleja en los estereotipos de la clase obrera: agresividad, fuerza, masculinidad, oposición a la disciplina y a la norma...

"Esto es debido a que el progreso educacional está controlado por medio del "justo" examen meritocrático de aquellas destrezas precisamente aportadas por el "capital cultural".

En la medida en que ésta es una valoración exacta del rol y de la importancia del título, apoya la visión de que es insensato para los chicos de la clase obrera confiar en los diplomas y en los certificados. Estos elementos no actúan para empujar a la gente hacia arriba -como en la versión oficial-sino que mantienen en su sitio a quienes ya han alcanzado la cumbre. En la medida en que el conocimiento siempre aparece sesgado y aportado a través de un significado de clase, el estudiante de la clase obrera debe, para empezar, superar su desventaja inicial de poseer la cultura de clase incorrecta y los descifradores educacionales incorrectos. Pocos pueden hacerlo. La clase nunca puede continuar. Sin embargo, sólo a través de un gran número de intentos se legitima la estructura de clases. La clase media disfruta de sus privilegios no en virtud de la herencia de nacimiento sino en virtud de una aparentemente demostrada mayor competencia y mérito. La negativa a competir, implícita en la cultura contraescolar es, por tanto, en este sentido, un acto radical: rechaza el complot para su supresión por medio del sistema educativo" (Willis, 1988: 155).

Finalmente, según este autor, para la clase obrera, el principal elemento de rechazo es el tipo de lenguaje que utilizan las clases acomodadas, mucho más abstracto e 
intelectualmente elaborado, frente al cual ellos plantean un uso mucho más vivo, pragmático y llano como modo de afirmación de pertenencia a su clase social.

Así lo confirma la siguiente cita:

"Para la clase obrera, en un sentido real, lo cultural es una batalla con el lenguaje. Esto no significa reducir lo cultural a un comportamiento anti-abstracto. Esto es postular, en parte, una manera antagónica de expresar lo abstracto y la vida mental centrada no en el sujeto individual, sino en el grupo: no en el lenguaje adquirido sino en la demostración vivida, la implicación directa y el control práctico.

Esto no significa negar la conciencia individual y el uso del lenguaje en su conexión dialéctica con la práctica de clase, sino que sugiere la posibilidad, en una sociedad de clases, de una forma asimétrica y distanciada de relación entre las dos. El lenguaje no es menos rico en la cultura contraescolar que en la cultura conformista - de hecho es mucho más incisivo y vital - pero no puede expresar, y por lo tanto no se usa de este modo, aquellas intuiciones mentales que desbordan el lenguaje adquirido. Los significados críticos que desbordan la fuerza de la creatividad en los grupos informales vuelven al grupo y a lo cultural para informar, reforzar y moldear muchas otras clases de prácticas físicas y estilísticas" (Willis, 1988: 149).

En síntesis, Bernstein afirma que las diferentes formas de habla crean y refuerzan en el usuario diferentes dimensiones de significación. Lo que hemos de preguntarnos es hasta qué punto las formas de habla de las diferentes clases sociales motivan la adquisición de significados diferentes más o menos cercanos a los que se valoran en el sistema educativo, y hasta qué punto esto condiciona el éxito en los resultados. Si analizamos la dimensión del significado, ¿hasta qué punto esta dimensión no guarda relación con la penetración de determinadas formas culturales vinculadas a la pertenencia a una clase social? y ¿cómo a través de estos mecanismos opera la reproducción social que vincula determinados usos del lenguaje con la posesión de unos significados relevantes y con una determinada estructura del poder y de la dominación en la sociedad?.

\section{DIMENSIONES DEL CÓDIGO LINGÜÍSTICO}

Para Bernstein, el tipo de lenguaje que posee cada uno de los individuos es consecuencia de una determinada forma de relación social que se establece en las diferentes clases sociales. Para explicar esta relación, Bernstein realiza una primera formulación del concepto de código en la que pretende vincular una determinada estructura lingüística, resultado de un determinado modo de utilizar el lenguaje, al desarrollo en los individuos de determinadas estrategias psicoafectivas en los individuos vinculadas al tipo de cultura de cada una de las clases sociales. No obstante, la definición que propone Bernstein es bastante más compleja, tal y como podemos observar en la siguiente cita: 
"Pueden definirse, a nivel lingüístico, en términos de probabilidad de predicción, para cualquier hablante, sobre qué elementos sintácticos utilizará para organizar el significado. En el caso del código elaborado, el hablante seleccionará entre una gama relativamente amplia de alternativas y, por tanto, la probabilidad de predicción será reducida. En el caso del código restringido, la cantidad de alternativas se encuentra enormemente limitada y la probabilidad de predicción del modelo se incrementa notablemente.

Anivel psicológico, se pueden diferenciar los códigos en función de cómo cada uno de ellos facilita (código elaborado) o inhibe (código restringido) la expresión simbólica de las intenciones bajo una forma verbal explícita. Sugiero que la conducta procesada por estos códigos desarrolla diferentes formas de orientación. Los propios códigos son funciones de una forma particular de relación social o, más generalmente, cualidades de la estructura social" (Bernstein,1989:85).

Es obvio que esta definición, plantẹada en términos de capacidad de predicción, es de difícil comprensión, y no resulta nada extraño que se rebata con el argumento de que presenta escaso valor científico. No obstante, la intuición que tiene Bernstein al relacionar los tres ámbitos merece atención. En este sentido, en el presente artículo se propone una reformulación de este concepto.

El código es una estructura que recoge tres dimensiones: una dimensión cultural vinculada a la pertenencia a una clase social, una dimensión conductual y una dimensión lingüística. ¿Qué queremos decir con los conceptos anteriores? Cuando hablamos de dimensión cultural nos referimos a las concepciones, creencias, valores, ideas y normas que hay en la sociedad, es decir, los contenidos culturales que forman la base cultural (la "conciencia" de todos los individuos) que pueden ser diferentes en los grupos sociales. Si nos referimos a la dimensión conductual, aludimos a los conceptos, normas y valores que rigen nuestra conducta, tales como, entre otros, los conceptos de autoridad, identidad, reflexividad o valores éticos, entre otros, que configuran modelos conductuales diferentes y que varían según la cultura. Evidentemente, los principios que rigen nuestra conducta no nacen en el vacío, sino que tienen un origen básicamente cultural. Estos conceptos configuran nuestra base moral y psicoafectiva.

Finalmente, tanto el contenido cultural como las conductas se plasman en una estructura lingüística determinada. En el proceso de socialización, la expresión lingüística es el eje fundamental que posibilita la adquisición de la cultura, la regulación de la conducta, y establece la base del aprendizaje del razonamiento a pesar de que éste no llegue a su máxima expresión hasta el período de las operaciones lógicas, es decir, cuando el individuo es capaz de razonar tanto la realidad que le envuelve, como el pasado y el futuro, a través de palabras y conceptos. Por tanto, mediante el proceso de aprendizaje de la lengua se forma no solamente la base lingüístico-conceptual y moral, sino también nuestra capacidad de razonamiento. Ahora bien, todos estos factores están condicionados por la posición que los individuos ocupan en la estructura social. 
En definitiva, cuando el niño aprende códigos lingüísticos específicos que regulan sus actos verbales, aprende los requisitos de su estructura social, que llega a ser el substrato de su experiencia. La estructura social se convierte en la realidad donde se produce el desarrollo psicológico del niño por medio de la configuración de sus actos de lenguaje, dado que la forma particular que adopta la relación social actúa selectivamente sobre lo que se dice (significados relevantes), cuándo se dice (contextos) y cómo se dice (formas de control). Las consecuencias de la forma que adopta la relación social se transmiten en términos de selección sintáctica y léxica, que constituyen procedimientos de construcción que guían al hablante en la preparación de su discurso y al oyente en su recepción. Así pues, diferentes formas de relaciones sociales pueden generar diferentes sistemas de lenguaje-uso o códigos lingüísticos.

Con posterioridad a la formulación del concepto de código, Bernstein propone una nueva formulación que tiene como objetivo explicar los mecanismos de reproducción del poder y del control en la sociedad. El código se convierte en un principio regulador adquirido de forma tácita, que selecciona e integra los significados relevantes para cada grupo social —es decir, lo que es significativo para cada uno de los grupos sociales-, las formas de realización lingüística - diferentes formas de lenguaje-uso- y los distintos contextos sociales que facilitan o inhiben determinados tipos de producción. Según este autor, las relaciones de clase derivadas de la división social del trabajo tienen una función distributiva que genera desigualdades en la distribución del poder y con los principios de control en la sociedad. Estas desigualdades se establecen a partir de la creación, distribución, reproducción y legitimación de los valores físicos y simbólicos propios de las clases dominantes, y por tanto, de los significados relevantes transmitidos en el modo de habla de cada uno de los grupos sociales. Este supuesto se hace muy evidente en el ámbito escolar, ámbito de reproducción y producción de la cultura oficial por excelencia.

En este sentido, según este autor, para poder garantizar la transmisión ideológica en la reproducción cultural, se establecen formas características de comunicación que posicionan a los individuos y los condicionan en sus relaciones tanto entre los diferentes grupos sociales, como en su seno. Las diferentes formas de comunicación se encuentran jerarquizadas, lo cual facilita la aparición de códigos dominantes $\mathrm{y}$ códigos dominados, $\mathrm{y}$ de formas de comunicación legítimas e ilegítimas. Las formas de comunicación legítimas se encuentran directamente vinculadas con los significados privilegiados - significados prioritarios de un contexto-y que privilegian - significados que confieren poder a quienes los utilizan.

A partir de este momento, Bernstein intenta aplicar este concepto macrosociológico a los diferentes contextos microsociológicos, es decir, aquellos que representan categorías especializadas en la división social del trabajo. El concepto código se aplica a ámbitos específicos. El grupo que domina los significados privilegiados en el interior de cada una de las categorías específicas disfruta del 
poder que este hecho le otorga y puede establecer los mecanismos de control, esto es, las formas de comunicación que le han de permitir permanecer en esta posición de poder. Es por ello que la distribución de las orientaciones de código depende de la distribución del poder creada por los principios que regulan la división social del trabajo.

\section{HACIA UN NUEVO MODELO DE INVESTIGACIÓN}

De lo que acabamos de exponer se desprenden numerosas dificultades. La primera de ellas es poner en relación la triple dimensión del concepto de código, es decir, la dimensión cultural de clase social, con la dimensión conductual y la estructura lingüística resultante. Los trabajos que se han realizado en el transcurso de estos años, ya sea por el mismo Bernstein $(1962,1963,1965,1966,1967) \mathrm{u}$ otros autores como Daniels (1988, 1995), Díaz (1990), Edwards (1994), Hasan (1993), Holland (1979, 1981), Sadovnik (1995), Tyler (1984, 1995), Wexler (1995) y, en nuestro propio país, Rodríguez Illera $(1986,1992)$ o Escofet $(1992,1994)$, han desarrollado aspectos muy parciales de la teoría. En este sentido, y a título indicativo, cabe destacar el trabajo realizado por Mayoral ${ }^{6}$ con la población escolar de $6^{\circ}$ curso de EGB de la ciudad de Lleida en el que se pone en relación la estructura social que se analiza a partir de las variables categoría ocupacional, barrio de residencia y capital cultural, con las producciones lingüísticas de los niños medidas en términos de inteligencia verbal y producción textual. La investigación se lleva a cabo con una muestra de 272 niños de la ciudad de Lleida, durante el curso 1992-1993, distribuidos proporcionalmente por barrios y que asisten a la escuela pública. Las conclusiones se centran en:

$1^{\circ}$. La ocupación no siempre mantiene una relación directa con el estatus sociocultural entendido en términos de capital cultural. No obstante, los niveles educativos de la población sí que mantienen una relación directa con la pertenencia a un estatus.

$2^{\circ}$. También se produce una relación estadísticamente significativa entre el estatus y la residencia, de manera que, a medida que ascendemos en el estatus, la residencia se sitúa en los barrios acomodados del interior de la ciudad.

$3^{\circ}$. La media de los coeficientes de inteligencia obtenidos después de haber agrupado los barrios es un factor que discrimina los diferentes grupos con una diferencia de 10 puntos. Este hecho corrobora la idea de que existe algún tipo de relación entre el coeficiente de inteligencia verbal y el estatus sociocultural.

El trabajo anterior observa la existencia de diferentes producciones lingüísticas $y$ diferentes coeficientes de inteligencia que pueden ser negativamente evaluados

\footnotetext{
${ }^{6}$ Este trabajo se desarrolla en dos publicaciones que referenciamos a continuación: Mayoral (1996), y en Mayoral (1998).
} 
desde la perspectiva escolar. Cuando esta evaluación negativa coincide con ámbitos de población escolar "negativamente" privilegiados desde la perspectiva social, las diferencias se han de interpretar en términos de desigualdad.

A partir de estas referencias proponemos un nuevo modelo de investigación que se fundamente:

$1^{\circ}$. En el análisis de la competencia lingüística que se manifiesta en el lenguaje escrito, dado que la investigación en la que se basa el presente artículo tiene como marco de análisis el contexto escolar, y es en este contexto en el que el lenguaje escrito adquiere todo su valor.

$2^{\circ}$. En considerar el capital cultural como el hecho más significativo en la configuración y el desarrollo de la competencia lingüística de los adolescentes, $y$ en distinguir en esta variable el nivel de estudios del padre y de la madre del resto de actividades que configuran el capital cultural familiar.

$3^{\circ}$. En partir de la base de que en una sociedad con una tradición cultural precaria los niveles de estudio alcanzados constituyen la clave esencial en la definición de capital cultural.

Así pues, en la investigación que se lleva a cabo en este artículo se intenta vincular el capital cultural familiar con el desarrollo de la competencia lingüística de los adolescentes. Nuestras hipótesis generales de trabajo son las siguientes:

$1^{\circ}$. Los hijos que pertenecen a familias con capital cultural elevado (entendido en términos de nivel de instrucción, consumos, hábitos y prácticas culturales) obtienen unos coeficientes de razonamiento verbal e índices de producción lingüística y textual superiores a la media.

$2^{\circ}$. El estatus sociocultural de la familia es más influyente que la clase de ocupación en el desarrollo de la competencia lingüística de los adolescentes.

De acuerdo con el modelo que acabamos de exponer, se desarrolló durante el curso 2000-2001 una investigación sobre el desarrollo del lenguaje de los adolescentes que cursaban primer curso de Educación Secundaria Obligatoria en todos los centros públicos y privados de la ciudad de Lleida. Los objetivos se centraban en conocer el nivel de influencia del capital cultural, especialmente, el nivel de estudios del padre y de la madre, sobre el desarrollo lingüístico de los adolescentes, así como valorar cuál de las variables (estatus sociocultural de la familia, o clase de ocupación) podía considerarse como más relevante en el desarrollo de la competencia lingüística (ver la investigación con más detalle en el Apéndice).

En lo que se refiere a los resultados obtenidos, nuestros análisis hacen evidente que la variable más substantiva en el desarrollo de la competencia lingüística de los niños es el nivel de estudios de los padres, fundamentalmente los estudios universitarios del padre y de la madre, tal como se refleja en la relación que se establece entre los índices lingüísticos y los niveles de estudios del padre y de la 
RIS

REVISTA INTERNACIONAL DE SOCIOLOGIA

No 41, MAY0-AGOST0, 2005

DOLORS MAYORAL ARQUÉ

madre. Las siguientes tablas muestran una relación estadísticamente significativa entre los/as niños/as que obtienen un índice lingüístico medio y alto y los padres que tienen estudios universitarios (en un $38,2 \%$ y en un $59,2 \%$, respectivamente). Aún es más nítida la relación que se establece entre los/as niños/as con un índice lingüístico alto y las madres con estudios universitarios $(53,2 \%)$. Por el contrario, no se obtiene ninguna relación estadística significativa entre los/as que tienen un índice lingüístico bajo y tanto el padre como la madre tienen, como nivel de formación más elevado, los estudios primarios o secundarios.

Tabla 1.

Índice de producción lingüistica/Nivel de estudios (padre)

\begin{tabular}{lcccccc}
\hline & Total & Sin estudios & $\begin{array}{c}\text { Estudios } \\
\text { primarios }\end{array}$ & $\begin{array}{c}\text { Estudios } \\
\text { secundarios }\end{array}$ & $\begin{array}{c}\text { Estudios } \\
\text { universitarios }\end{array}$ & ns/nc \\
\hline $\mathrm{N}^{\circ}$ individuos & 440 & 6 & 259 & 61 & 76 & 38 \\
Bajo & 6,4 & 16,7 & 8,1 & 3,3 & 2,6 & 5,3 \\
Medio & 51,1 & 66,7 & 52,5 & 52,5 & $\mathbf{3 8 , 2}$ & 63,2 \\
Alto & 42,5 & 16,7 & 39,4 & 44,3 & $\mathbf{5 9 , 2 +}$ & 31,6 \\
Media & 52,28 & 47,83 & 50,94 & 53,44 & 57,25 & 50,32 \\
d.t. & 16,70 & 19,2 & 16,98 & 15,25 & 16,26 & 15,91 \\
\hline
\end{tabular}

Tabla 2.

Índice de producción lingüistica/Nivel de estudios (madre)

\begin{tabular}{lcccccc}
\hline & Total & Sin estudios & $\begin{array}{c}\text { Estudios } \\
\text { primarios }\end{array}$ & $\begin{array}{c}\text { Estudios } \\
\text { secundarios }\end{array}$ & $\begin{array}{c}\text { Estudios } \\
\text { universitarios }\end{array}$ & $\mathrm{ns} / \mathrm{nc}$ \\
\hline $\mathrm{N}^{\circ}$ individuos & 440 & 6 & 287 & 46 & 77 & 24 \\
Bajo & 6,4 & 16,7 & 5,6 & 6,5 & 1,3 & 4,2 \\
Medio & 51,1 & - & 30,3 & 37 & 45,5 & $75+$ \\
Alto & 42,5 & 33,3 & 49,5 & 52,2 & $\mathbf{5 3 , 2 +}$ & $20,8-$ \\
Media & 52,28 & 50 & 14,6 & $4,3-$ & 55,81 & 49,13 \\
d.t. & 16,70 & 5 & 7,61 & 8,74 & 15,43 & 15,05 \\
\hline
\end{tabular}

Una relación similar, aunque más débil, se establece entre los índices de producción lingüística y textual y los índices culturales familiares. Por una parte, a 
indices de producción lingüística altos les corresponden índices culturales medios en un 50,3\%. Por otra, los índices de producción textual que requieren, además, habilidades lingüísticas, la aplicación de estrategias de razonamiento que les han de permitir ordenar y ligar las secuencias verbales. Observamos que, junto a la relación anterior, se manifiesta una relación estadísticamente significativa entre los que poseen un índice de producción textual muy bajo y al mismo tiempo las familias obtienen un índice cultural también bajo.

Tabla 3.

Índice de producción lingüistical Índice cultural

\begin{tabular}{lccccccc}
\hline & Total & Muy bajo & Bajo & Media & Alto & Muy alto & $\mathrm{m} /$ d.t. \\
\hline No individuos & 440 & 49 & 209 & 153 & 28 & 1 & 440 \\
Bajo & 6,4 & 10,2 & 7,7 & 3,9 & 3,6 & - & 6,4 \\
Medio & 51,1 & 59,2 & 53,6 & 45,8 & 50,0 & - & 51,1 \\
Alto & 42,5 & 30,6 & 38,8 & $\mathbf{5 0 , 3 +}$ & 46,4 & 100,0 & 42,5 \\
Media & 52,28 & 47,39 & 51,10 & 55,01 & 54,25 & 67,00 & 52,28 \\
d.t. & 16,70 & 18,15 & 16,44 & 16,50 & 14,98 &, 00 & 16,70 \\
\hline
\end{tabular}

Tabla 4.

Índice de producción textual/Índice cultural

\begin{tabular}{lccccccc}
\hline & Total & Muy bajo & Bajo & Media & Alto & Muy alto & m/, d.t. \\
\hline No individuos & 440 & 49 & 209 & 153 & 28 & 1 & 440 \\
Muy bajo & 20,9 & $44,9+$ & 23,9 & $\mathbf{1 1 , 1 -}$ & 10,7 & - & 20,9 \\
Bajo & 35,7 & 32,7 & 36,4 & 35,9 & 32,1 & 100,0 & 35,7 \\
Medio & 32,0 & $\mathbf{1 6 , 3 -}$ & 30,6 & 37,3 & 42,9 & - & 32,0 \\
Elevado & 11,4 & 6,1 & 9,1 & $\mathbf{1 5 , 7 +}$ & 14,3 & - & 11,4 \\
Media & 82,23 & 59,18 & 78,18 & 93,20 & 93,57 & 60,00 & 82,23 \\
d.t. & 43,00 & 43,20 & 42,73 & 40,27 & 40,39 &, 00 & 43,00 \\
\hline
\end{tabular}

Si persistimos en nuestro análisis para poder ver cuál es el peso de las diferentes variables sociológicas en el desarrollo lingüístico, constatamos como los padres 
que leen diariamente influyen en un $49,4 \%$ de los/as niños/as que obtienen un Índice lingüístico alto. Contrariamente, el $32 \%$ de los niños cuyas madres leen algunas veces obtienen un índice lingüístico alto.

Tabla 5 .

Índice lingüistico/Frecuencia de lectura (padre)

\begin{tabular}{lccccccc}
\hline & Total & $\begin{array}{c}\text { Cada } \\
\text { día }\end{array}$ & $\begin{array}{c}\text { Algunos días } \\
\text { a la semana }\end{array}$ & $\begin{array}{c}\text { Un día a } \\
\text { la semana }\end{array}$ & $\begin{array}{c}\text { Algunas } \\
\text { veces }\end{array}$ & $\begin{array}{c}\text { No lee } \\
\text { nunca }\end{array}$ & ns/nc \\
\hline $\mathrm{N}^{\circ}$ individuos & 440 & 164 & 119 & 12 & 94 & 12 & 39 \\
Bajo & 6,4 & 4,3 & 5,9 & 25 & 6,4 & 8,3 & 10,3 \\
Medio & 51,1 & 46,3 & 47,1 & 33,3 & 59,6 & 75 & 61,5 \\
Alto & 42,5 & $49,4+$ & 47,1 & 41,7 & 34 & 16,7 & 28,2 \\
Media & 52,28 & 54,55 & 53,54 & 50,17 & 49,77 & 46,58 & 47,38 \\
d.t. & 16,70 & 16,59 & 15,79 & 22,16 & 16,15 & 17,90 & 17,13 \\
\hline
\end{tabular}

Tabla 6.

Índice lingüistico/Frecuencia de lectura (madre)

\begin{tabular}{lccccccc}
\hline & Total & $\begin{array}{c}\text { Cada } \\
\text { día }\end{array}$ & $\begin{array}{c}\text { Algunos días } \\
\text { a la semana }\end{array}$ & $\begin{array}{c}\text { Un día a } \\
\text { la semana }\end{array}$ & $\begin{array}{c}\text { Algunas } \\
\text { veces }\end{array}$ & $\begin{array}{c}\text { No lee } \\
\text { nunca }\end{array}$ & ns/nc \\
\hline $\mathrm{N}^{\circ}$ individuos & 440 & 175 & 147 & 13 & 79 & 6 & 19 \\
Bajo & 6,4 & 4 & 6,1 & 15,4 & 10,1 & - & 10,5 \\
Medio & 51,1 & 48,6 & 47,6 & 46,2 & 58,2 & 66,7 & 68,4 \\
Alto & 42,5 & 47,4 & 46,3 & 38,5 & $31,6-$ & 33,3 & 21,1 \\
Media & 52,28 & 54,06 & 53,35 & 50,38 & 47,97 & 55,17 & 45,74 \\
d.t. & 16,70 & 15,81 & 16,82 & 18,68 & 17,25 & 16,04 & 17,04 \\
\hline
\end{tabular}

En lo que se refiere a la relación entre el índice lingüístico y la clase de ocupación del padre, no se obtiene una relación significativa. Contrariamente, en las madres de clase media, muy significativamente en las de la nueva clase media, sí se observa una relación estadísticamente significativa entre tener 
unos índices lingüísticos medios y altos y pertenecer a la nueva clase media ( $41,0 \%$ y $54,2 \%$, respectivamente). Esta relación adquiere más fuerza cuando se observa la relación entre los índices de producción textual y la clase de ocupación de la madre, muy especialmente, entre las madres de la nueva clase media (38,9\%).

En definitiva, la clase de ocupación del padre es escasamente relevante en el desarrollo lingüístico de los niños, hecho que puede atribuirse a la escasa presencia de los padres en el hogar y, todavía, a la precaria dedicación en la educación de los hijos de un importante sector de la población masculina. No obstante, no basta con levantar el dedo para señalar a los culpables. La falta de políticas familiares adecuadas fomenta la supervivencia forzosa de algunos de estos hábitos. Sin embargo, también es necesario considerar la necesidad de trabajar muchas horas, e incluso desarrollar dos trabajos, para poder hacer frente a los excesivos gastos del hogar. Los datos que corroboran las afirmaciones posteriores se pueden observar en las tablas siguientes (tabla 7 y 8 ).

Si nos preguntamos qué relación mantiene el estatus sociocultural de la familia con el desarrollo de la competencia lingüística de los niños, constatamos relaciones similares a las anteriores. A índices de producción lingüística bajos y medios les corresponden estatus socioculturales bajos en un $13,3 \%$, y medios en un $61,4 \%$. En el caso de índices de producción lingüística medios y altos, se corresponden con estatus altos o medios-altos en un 53,3\%. La relación que se establece entre los índices de producción textual y el estatus es más significativa en las posiciones bajas, tal y como se refleja en las tablas 9 y 10.

Tabla 7.

Índice lingüistico/Clase de ocupación (madre)

\begin{tabular}{lcccccccc}
\hline & Total & $\begin{array}{c}\text { Clase } \\
\text { obrera }\end{array}$ & $\begin{array}{c}\text { Vieja } \\
\text { clase } \\
\text { media }\end{array}$ & $\begin{array}{c}\text { Nueva } \\
\text { clase } \\
\text { media }\end{array}$ & $\begin{array}{c}\text { Clases } \\
\text { medias } \\
\text { técnicas }\end{array}$ & $\begin{array}{c}\text { Clase } \\
\text { alta }\end{array}$ & Inactivos & ns/nc \\
\hline $\mathrm{N}^{\circ}$ individuos & 440 & 4 & 51 & 144 & 37 & 31 & 141 & 32 \\
Bajo & 6,4 & - & 15,7 & 4,9 & 5,4 & - & 5,7 & 9,4 \\
Medio & 51,1 & 50,0 & 49,0 & $41,0-$ & 62,2 & 61,3 & 55,3 & 59,4 \\
Alto & 42,5 & 50,0 & 35,3 & $54,2+$ & 32,4 & 38,7 & 39,0 & 31,3 \\
Media & 52,28 & 58,50 & 48,53 & 55,25 & 50,24 & 51,58 & 52,05 & 48,19 \\
d.t. & 16,70 & 13,44 & 18,17 & 15,84 & 18,33 & 16,87 & 16,07 & 17,64 \\
\hline
\end{tabular}


Tabla 8.

Índice de producción textual/ Clase de ocupación (madre)

\begin{tabular}{lcccccccc}
\hline & Total & $\begin{array}{c}\text { Clase } \\
\text { obrera }\end{array}$ & $\begin{array}{c}\text { Vieja } \\
\text { clase } \\
\text { media }\end{array}$ & $\begin{array}{c}\text { Nueva } \\
\text { clase } \\
\text { media }\end{array}$ & $\begin{array}{c}\text { Clases } \\
\text { medias } \\
\text { técnicas }\end{array}$ & $\begin{array}{c}\text { Clase } \\
\text { alta }\end{array}$ & Inactivos & ns/nc \\
\hline $\mathrm{N}^{0}$ individuos & 440 & 4 & 51 & 144 & 37 & 31 & 141 & 32 \\
Muy bajo & 20,9 & - & $33,3+$ & $13,9-$ & 27,0 & 19,4 & 21,3 & 28,1 \\
Bajo & 35,7 & 50,0 & 23,5 & 36,8 & 32,4 & 29,0 & 39,7 & 40,6 \\
Medio & 32,0 & 25,0 & 23,5 & $\mathbf{3 8 , 9 +}$ & 32,4 & 38,7 & 27,0 & 31,3 \\
Elevado & 11,4 & 25,0 & $19,6+$ & 10,4 & 8,1 & 12,9 & 12,1 & - \\
Media & 82,23 & 100,00 & 80,78 & 86,53 & 78,92 & 89,68 & 80,43 & 67,50 \\
d.t. & 43,00 & 37,42 & 53,51 & 39,14 & 42,15 & 41,59 & 43,59 & 39,59 \\
\hline
\end{tabular}

Tabla 9.

Índice de producción lingüisticalestatus

\begin{tabular}{lcccccc}
\hline & Total & Alto & Medio-alto & Medio & Medio-bajo & Bajo \\
\hline $\mathrm{N}^{\circ}$ individuos & 440 & 83 & 90 & 88 & 96 & 83 \\
Bajo & 6,4 & 2,4 & 2,2 & 4,5 & 9,4 & $\mathbf{1 3 , 3 +}$ \\
Medio & 51,1 & 48,2 & 44,4 & 52,3 & 50,0 & $\mathbf{6 1 , 4 +}$ \\
Alto & 42,5 & 49,4 & $\mathbf{5 3 , 3 +}$ & 43,2 & 40,6 & $25,3-$ \\
Media & 52,28 & 55,25 & 56,41 & 52,75 & 50,89 & 45,95 \\
d.t. & 16,70 & 16,27 & 15,45 & 15,75 & 17,11 & 17,20 \\
\hline
\end{tabular}

Tabla 10.

Índice de producción textual/estatus

\begin{tabular}{lcccccc}
\hline & Total & Alto & Medio-alto & Medio & Medio-bajo & Bajo \\
\hline No individuos & 440 & 83 & 90 & 88 & 96 & 83 \\
Muy bajo & 20,9 & 14,5 & $\mathbf{1 2 , 2 -}$ & $\mathbf{9 , 1 -}$ & $\mathbf{3 1 , 3 +}$ & $37,3+$ \\
Bajo & 35,7 & 30,1 & 33,3 & 42 & 34,4 & 38,6 \\
Medio & 32,0 & 41 & 37,8 & 35,2 & 26 & $20,5-$ \\
Elevado & 11,4 & 14,5 & 16,7 & 13,6 & 8,3 & $3,6-$ \\
Media & 82,23 & 93,25 & 94 & 91,59 & 71,67 & 60,72 \\
d.t. & 43,00 & 40,88 & 41,38 & 36,73 & 44,74 & 40,66 \\
\hline
\end{tabular}

128 


\section{CONCLUSIONES}

En síntesis, se observa que el nivel de estudios alcanzado por los padres es especialmente significativo en el desarrollo de la competencia lingüística de los adolescentes, entendida ésta en términos de conocimiento lingüístico y nivel de estructuración y coherencia cognitiva. Otros dos aspectos que merecen ser objeto de atención se refieren a:

a) la influencia que los padres que leen diariamente ejercen sobre el desarrollo de la competencia lingüística de los hijos.

b) las madres que realizan ocupaciones consideradas como pertenecientes a la nueva clase media desarrollan, a su vez, una influencia positiva en la evolución de las competencias lingüísticas de los hijos. En lo que se refiere a este último factor, cabe destacar su influencia tanto a nivel verbal, como a nivel de razonamiento cognitivo.

La relación que se establece entre el estatus sociocultural y el desarrollo de la competencia comunicativa de los adolescentes sólo resulta significativa en aquellos casos en los que se relaciona un estatus sociocultural bajo con un desarrollo de la competencia lingüística también bajo.

Además de estas conclusiones se aprecia una serie de matices que plantean nuevos interrogantes: ¿A qué podemos atribuir el hecho de que los padres con un elevado nivel de estudios y lectores frecuentes tengan una mayor influencia que las madres en su misma posición en el desarrollo lingüístico de los adolescentes? ¿Puede tratarse de modelos culturales de razonamiento sensiblemente diferentes, en función de los modelos de socialización de género? (Por ejemplo, planteamiento de determinados temas de conversación, modelos de razonamiento, intereses diferentes según el género...). ¿Hasta qué punto los modelos masculinos de razonamiento se encuentran más próximos al tipo de cultura escolar? ¿Podrían ser susceptibles de analizarse las anteriores desigualdades en términos de códigos de género? ¿Hasta qué punto unos pueden ser más influyentes que otros en el contexto escolar?

Por otra parte, debemos prestar atención a los resultados obtenidos en el caso de las madres que desarrollan un trabajo que requiere determinadas habilidades sociales y cognitivas (nueva clase media). Podemos preguntarnos si la incorporación y la consolidación de la mujer en el mundo laboral (el desarrollo implícito de determinadas habilidades que conlleva) pueden ser especialmente significativas en el cambio de estrategias de razonamiento. Esta conclusión nos permite preguntarnos acerca de las condiciones que deben darse para que los códigos utilizados por ambos géneros puedan ser igualmente influyentes en el desarrollo lingüístico de los adolescentes.

Otras apreciaciones pueden realizarse a partir del análisis de los datos obtenidos. Observamos resultados diferentes entre los índices de producción lingüística y el estatus, y los índices de producción textual y el estatus en los diferentes grupos. 


\section{RIS}

La diferencia que se establece entre unos índices y otros se encuentra en que:

a) Los índices de producción lingüística mantienen una relación significativa con los que pertenecen a un estatus medio-alto.

b) No se establece la relación anterior entre los índices de producción textual y los niveles de estatus medios o medio-altos.

¿A qué factores puede atribuirse la aparente contradicción anterior? En primer lugar, los índices de producción lingüística se refieren al dominio del vocabulario y a la construcción de estructuras sintácticas complejas, mientras que los índices de producción textual requieren que las estructuras se encuentren debidamente secuenciadas y estructuradas. En otras palabras, una cosa es producir una oración y otra es producir un texto coherente y estructurado. Los índices de producción lingüística y textual se refieren a actos de lenguaje y raciocinio diferenciados.

¿Cómo puede explicarse esta diferencia? Cabe suponer que determinados grupos de familias pueden cultivar actividades culturales y sociales que faciliten el desarrollo de las habilidades comunicativas. Ahora bien, dicho desarrollo no siempre se ve acompañado de la aplicación de estrategias cognitivas cuya base se encuentra en un razonamiento coherente y jerarquizado.

En definitiva, ¿en qué medida nuestros hallazgos contribuyen a reforzar la teoría bernsteniana de los códigos? Ciertamente, las conclusiones que se obtienen ponen de manifiesto, una vez más, la existencia de algún tipo de relación entre las culturas que se desarrollan en determinados grupos sociales "negativamente privilegiados"y el nivel de desarrollo de determinadas habilidades lingüísticas y cognitivas. Sin embargo, dicha afirmación debe acompañarse de toda una serie de precisiones:

1. La investigación desarrollada no abarca todos los aspectos del concepto código en su totalidad. Tiene limitaciones en lo que se refiere a la aplicación estricta del mismo concepto, así como en la limitación expresada por la muestra. Necesitamos más investigaciones en otros contextos y con otras variables para ver si los resultados se repiten.

2. En nuestra investigación no se ha utilizado el paralelismo clase social/ desarrollo lingüístico, sino que se han utilizado otras variables como el nivel de estudios del padre y de la madre, el índice de capital cultural familiar (medido a partir de los índices de actividades realizadas por el padre y por la madre) y la clase de ocupación de ambos cónyuges.

3. Las pruebas relacionadas con el desarrollo de la competencia comunicativa, aparte de ser consideradas únicamente en su nivel de desarrollo escrito, se han planteado con una doble finalidad: el conocimiento de vocabulario, y la capacidad para redactar textos coherentes y debidamente estructurados como medida de la capacidad de raciocinio. 
Las observaciones anteriores nos permiten concluir que el nivel de estudios de los padres es un factor fundamental en el desarrollo de las habilidades lingüísticas y cognitivas de los adolescentes. Sin embargo, a pesar de la importancia de los resultados, observamos que se producen excepciones que nos inducen a pensar que no se trata de una relación determinista ${ }^{7}$. Ello nos remite a la propia concepción de Bernstein cuando define sus investigaciones como una sociología de las transmisiones educativas.

Otro aspecto que debemos destacar en la construcción de un marco analítico de la teoría de los códigos es la importancia de la variable capital cultural. En este sentido, la aportación de Bourdieu nos parece crucial y muy acorde con la presencia en nuestras sociedades de nuevas desigualdades culturales basadas en criterios de capital cultural y niveles educativos alcanzados.

Esta nueva relación vendría a explicar dos supuestos anteriormente indicados por estos autores:

a) El capital cultural familiar, muy especialmente el nivel de estudios alcanzado por los padres, tiene una influencia considerable en el desarrollo de la competencia lingüística de los/as hijos/as. De hecho, Bourdieu avanza en La Distinción (1991) la presencia de un modelo circular en el que el nivel de estudios familiar sitúa a los hijos/as en condiciones de obtener un grado de éxito escolar más elevado. Estos grupos sociales - agrupados bajo el nombre de nueva clase media- son proclives a invertir más en educación, a diferencia de aquellas fracciones de clase alta o media-alta, que, poseedores de capital económico, disponen de un margen más amplio de libertad en las elecciones, dado que tienen garantizada la base de su reproducción social.

b) Sin embargo, la propensión de la familia a invertir en capital cultural no explica todo el proceso por medio del cual una fracción de clase se adapta con más facilidad al contexto escolar. Tal como intuyó Bernstein (1975: 123-126) este grado más elevado de éxito puede ser consecuencia del desarrollo de unas afinidades ideológicas entre estos grupos sociales y los profesionales que trabajan en el ámbito del control simbólico, bien sea desde la esfera del Estado como burócratas en los diseños de los planes de estudio, currículo y estrategias pedagógicas, o bien desde la esfera privada a través de las editoriales y de los medios de comunicación de masas.

Los resultados anteriores nos plantean la introducción de nuevas variables en el análisis del concepto de código que, finalmente, nos remiten a las estrategias

\footnotetext{
${ }^{7}$ En esta investigación se detecta la presencia de un reducido numero de individuos que no responden a la relación anterior porque, o bien pertenecen a familias con un nivel de estudios bajo, o bien pertenecen a familias con un elevado nivel de estudios. Estos adolescentes no obtienen resultados positivos, especialmente, en lo que se refiere a los índices de producción textual. La no presencia de la relación anterior puede ser debida al coeficiente de inteligencia, o bien al tipo de relación educativa que se establece en el seno de la familia, variables que no han podido tenerse en cuenta en dicha investigación.
} 
sobre la transmisión del poder y del control social. Las diferencias en la producción de códigos lingüísticos pueden estar debidas no única y exclusivamente a factores de clase social, sino también a factores educativos, a niveles de estudios alcanzados y, aún más, a la presencia de códigos de género. También debería analizarse la influencia de las políticas educativas en la definición de las estrategias curriculares para conocer el grado de acercamiento y disensión entre determinados grupos sociales especializados en el ámbito de la producción y reproducción culturales y las culturas de la sociedad. Si de lo que se trata es de evitar el elevado fracaso educativo, al tiempo que se potencian los niveles culturales de la población, deben realizarse muchos procesos de análisis que no pueden permanecer al margen de las características culturales y lingüísticas de los diferentes grupos sociales.

\section{REFERENCIAS BIBLIOGRÁFICAS}

BERGER, P. y TH. LUCKMANN (1988), La construcció social de la realitat. Un tractat de sociologia del coneixement, Barcelona, Herder.

BERNSTEIN, B. (1988), Clases, códigos y control. Hacia una teoria de las transmisiones educativas, vol II, ed. Akal, pp. 109-144.

(1989), Clases, códigos y control. Estudios teóricos para una sociología del lenguaje, vol. I, Madrid, Akal.

(1993), La estructura del discurso pedagógico, Madrid, Morata.

(1998), Pedagogia, control simbólico e identidad, Madrid, Morata.

(1999), "Vertical and horizontal discourse: An essay", British Journal of Sociology of Education, vol. 20, pp.157-173.

BERNSTEIN, B. y J. SOLOMÓN (1999), "Pedagogy, Identity and the Construction of a Theory of Symbolic Control: Basil Bersntein questioned by Joseph Solomon", British Journal of Sociology of Education, vol. 20, pp. 264-269.

BOURDIEU, P. (1985), ¿Qué significa hablar? Economia de los intercambios lingüisticos, Madrid, Akal.

(1991), La distinción. Criterio y bases sociales del gusto, Madrid, Taurus.

BRUNER, J. (1991), Actos de significado, Madrid, Alianza.

CACOUAULT, M. y F. OEUVRARD (2001), Sociologie de l'éducation, (repères), París, La Découverte.

CASTELLS, M. (1995), La ciudad informacional, Madrid, Alianza. 
CROMPTON, R. (1994), Clase y estratificación. Una introducción a los debates actuales, Madrid, Tecnos.

ESPING-ANDERSEN, G. (2000), Fundamentos sociales de las economias postindustriales, Barcelona, Ariel.

GIL CLAVO, E. (1993), La era de las lectoras: el cambio cultural de las mujeres españolas, Madrid, Ministerio de Asuntos Sociales, Instituto de la Mujer.

GIMENO SACRISTÁN, J. (2001), Educar y convivir en la cultura global, Madrid, Morata.

GINER, S. (1996), Sociología, Barcelona, Península.

GIRÓ, P. (1997), Desigualtats socials als barris de Lleida. Segregació urbana i educació, Pagès.

KOZULIN, A. (1994), La psicología de Vygotski, Madrid, Alianza.

LAVOB, W. (1979), Sociolinguistique, París, Les éditions de minuit.

MAYORAL, D. (1996), "Diferenciación y desigualdad: cuando las diferencias culturales se convierten en desigualdades sociales“, Revista de Educación, $\mathrm{n}^{\circ} 311$, pp. 159-183.

(1998), El lenguaje: diferencias culturales y desigualdades sociales, Lleida, Pagès.

MAYORAL, D. y A. GIRÓ (2001), Percepcions, pràctiques i dimensions dels sectors culturals a Lleida, Lleida, Pagès.

MOLINA LUQUE, J.F. (2002), Sociología de la educación intercultural: vias alternativas de investigación y debate, Buenos Aires, Lumen.

MORAIS, A., I. NEVES, B. DAVIES y H. DANIELS (2001), Towards a Theory of Pedagogy. The contribution of Basil Bernstein to Research, Nueva York, Peter Lang.

MULLER, J., B. DAVIES y A. MORAIS (2004), Reading Bersntein, Researching Bersntein, Londres, Rouletge/Falmer.

NASH, R. (1999), "Bourdieu, "Habitus", and Educational Research: is it all worth the candle?", British Journal of Sociology of Education, $\mathrm{n}^{\circ} 20$, pp. 175-187.

(1999), "Realism in the Sociology iof Education:"explain social differences in attainment", British Journal of Sociology of Education, $\mathrm{n}^{\mathrm{O}}$ 20, pp. 107-125.

OLIN WRIGHT, E. (1995), "El análisis de clase de la pobreza", en Carabaña, J. (ed.), Desigualdad $y$ clases sociales. Un seminario en torno a Erik O. Wright, Madrid, Fundación Argentaria, Visor, pp. 133-151.

PÉREZ GÓMEZ, A.I. (2000), La cultura escolar en la sociedad neoliberal, Madrid, Morata.

POWER, S. (2000), "Educational Pathways into the Middle Class(es)", British Journal of Sociology of Education, $\mathrm{n}^{\circ} 21, \mathrm{pp} .133-145$. 


\section{RIS}

SAMPER, Ll. y D. MAYORAL (1998), "Homogamia educativa y capital cultural”, Papers, $\mathrm{n}^{\circ}$ 54, pp. 139-155.

SIMONE, R. (2000), La Tercera Fase. Formas de saber que estamos perdiendo, Santafé de Bogotá, Taurus.

STERNBERG, R. y D. DETTERMAN (1992), ¿Qué es la inteligencia? Enfoque actual de su naturaleza y definición, Madrid, Pirámide.

STUBBS, M. (1982), El llenguatge i l'escola. Cap a una anàlisi sociolingüistica en educació, Barcelona, Editorial, 62.

TEZANOS, J. F. (2001), La sociedad dividida. Estructuras de clases y desigualdades en las sociedades tecnológicas, Madrid, Biblioteca Nueva.

VARELA, J. (1991), “Una reforma educativa para las nuevas clases medias”, en Fernández Enguita, M. (ed.) Sociología de la Educación, Barcelona, Ariel.

VVAA (2004), "Special Issue: Pierre Bourdieu's Sociology of Education: the Theory of Practice and the Practice and Theory", British Journal of Sociology of Education, $\mathrm{n}^{\circ} 25$.

WILLIS, P. (1988), Aprendiendo a trabajar, Madrid, Akal.

\section{APÉNDICE}

El estudio se ha circunscrito a los/las adolescentes que han cursado primer curso de Educación Secundaria Obligatoria en los centros públicos y privados de la ciudad de Lleida durante el curso 2000-2001. La unidad de análisis sociológico ha sido el hogar, aunque en determinadas variables se ha distinguido entre el padre y la madre. La unidad de análisis lingüístico han sido los niños y niñas de primer curso de Educación Secundaria Obligatoria.

La muestra corresponde a 440 individuos y representa a la totalidad de niños y niñas que han cursado durante el curso 2000-2001 primer curso de Educación Secundaria Obligatoria sobre un total de 1492, según datos del Consell Escolar Municipal para el curso 2000-2001. La muestra se ha calculado por medio de la aplicación de la fórmula $n=4 p . q . N$

$$
\mathrm{e} 2(\mathrm{~N}-1)+4 p .9
$$

El nivel de confianza de la muestra es de un sigma 2 con un grado de error de $+-4 \%$.

Como instrumentos de medida se han utilizado un cuestionario sociológico, pruebas de razonamiento verbal del test TEA 2 y un dibujo que ha inspirado las producciones textuales. 
Se han introducido nuevas medidas de la producción lingüística que han consistido en la elaboración de un índice de producción lingüística y un índice de producción textual.

El cuestionario sociológico incluye veintidós ítems que nos dan información sobre las variables siguientes: datos sociodemográficos, niveles de instrucción, uso de la lengua catalana y castellana, posición en el mercado de trabajo, equipamientos culturales, consumos, hábitos y prácticas culturales.

Los índices se han elaborado de la manera siguiente:

Índice de producción lingüistica: número de substantivos, adjetivos, verbos, oraciones simples, oraciones coordinadas y oraciones subordinadas.

El valor máximo que puede alcanzar un individuo es de 85 puntos. La distribución del número de casos en una curva normalizada se ha calculado sobre las puntuaciones resultantes de los mismos índices. Este procedimiento ha permitido distribuir los intervalos tal y como se presenta a continuación:

\section{Valor obtenido}

entre 1 y 17 puntos

entre 18 y 34 puntos

entre 35 y 51 puntos

entre 52 y 68 puntos

entre 69 y 85 puntos

\author{
Clasificación índice de producción lingüistica \\ índice de producción lingüística muy bajo \\ índice de producción lingüística bajo \\ índice de producción lingüística medio \\ índice de producción lingüística alto \\ índice de producción lingüística muy alto
}

Índice de producción textual: se ha elaborado a partir de las variables coherencia, cohesión y adecuación textual.

El valor máximo que puede obtener un individuo es de 136 puntos. La distribución del número de casos en una curva normalizada se ha calculado sobre las puntuaciones resultantes de los mismos índices. Este procedimiento ha permitido distribuir los intervalos tal y como se presenta a continuación.

\author{
Valor obtenido \\ entre 1 y 45 puntos \\ y 90 puntos \\ entre 91 y 135 puntos
}

\section{Clasificación índice de producción textual} indice de producción textual muy bajo entre 46 índice de producción textual bajo índice de producción textual medio más de 136 índice de producción textual alto 